\title{
Accuracy of mean arterial pressure and blood pressure measurements in predicting pre-eclampsia: systematic review and meta-analysis
}

'Department of General Practice, Academic Medical Center, Meibergdreef 15, 1100 DD, Amsterdam, Netherlands

${ }^{2}$ Horten Center, University of Zurich, Switzerland

${ }^{3}$ Department of Obstetrics and Gynaecology, Academic Medical Center, Amsterdam, Netherlands

${ }^{4}$ Department of Obstetrics and Gynaecology, St Elisabeth Hospital, Tilburg, Netherlands

${ }^{5}$ Department of Obstetrics and Gynaecology, Birmingham Womens Hospital, Birmingham

Correspondence to: I S Cnossen j.s.cnossen@amc.uva.nl

doi:10.1136/bmj.39540.522049.BE

\author{
Jeltsje S Cnossen, research fellow, ${ }^{1}$ Karlijn C Vollebregt, research fellow, ${ }^{3}$ Nynke de Vrieze, medical \\ student, ${ }^{1}$ Gerben ter Riet, associate professor, ${ }^{2}$ Ben W J Mol, professor, ${ }^{3}$ Arie Franx, consultant, ${ }^{4}$ \\ Khalid S Khan, professor, ${ }^{5}$ Joris A M van der Post, professor ${ }^{3}$
}

\section{ABSTRACT}

Objective To determine the accuracy of using systolic and diastolic blood pressure, mean arterial pressure, and increase of blood pressure to predict pre-eclampsia. Design Systematic review with meta-analysis of data on test accuracy.

Data sources Medline, Embase, Cochrane Library, Medion, checking reference lists of included articles and reviews, contact with authors.

Review methods Without language restrictions, two reviewers independently selected the articles in which the accuracy of blood pressure measurement during pregnancy was evaluated to predict pre-eclampsia. Data were extracted on study characteristics, quality, and results to construct $2 \times 2$ tables. Summary receiver operating characteristic curves and likelihood ratios were generated for the various levels and their thresholds.

Results 34 studies, testing 60599 women (3341 cases of pre-eclampsia), were included. In women at low risk for pre-eclampsia, the areas under the summary receiver operating characteristic curves for blood pressure measurement in the second trimester were $0.68(95 \%$ confidence interval 0.64 to 0.72 ) for systolic blood pressure, 0.66 (0.59 to 0.72 ) for diastolic blood pressure, and 0.76 (0.70 to 0.82 ) for mean arterial pressure. Findings for the first trimester showed a similar pattern. Second trimester mean arterial pressure of $90 \mathrm{~mm} \mathrm{Hg}$ or more showed a positive likelihood ratio of 3.5 (95\% confidence interval 2.0 to 5.0 ) and a negative likelihood ratio of 0.46 ( 0.16 to 0.75$)$. In women deemed to be at high risk, a diastolic blood pressure of $75 \mathrm{~mm} \mathrm{Hg}$ or more at 13 to 20 weeks' gestation best predicted pre-eclampsia: positive likelihood ratio 2.8 (1.8 to 3.6), negative likelihood ratio 0.39 ( 0.18 to 0.71 ). Additional subgroup analyses did not show improved predictive accuracy.

Conclusion When blood pressure is measured in the first or second trimester of pregnancy, the mean arterial pressure is a better predictor for pre-eclampsia than systolic blood pressure, diastolic blood pressure, or an increase of blood pressure.

\section{INTRODUCTION}

Pre-eclampsia is an important disorder of pregnancy, with potentially severe consequences for mother and child. ${ }^{12}$ The frequency of pre-eclampsia varies between $2 \%$ and $7 \%$ in healthy nulliparous women..$^{3-5}$ In preeclampsia the interaction between the placenta and maternal constitution is influenced by genetic and environmental factors, causing a hypertensive inflammatory response. ${ }^{6}$ The gestosis is associated with low birth weight and preterm delivery but can also develop at term, during labour, or even post partum. ${ }^{2}$ It has been shown that women destined to develop preeclampsia have higher mean arterial pressures in the first and second trimester and even before pregnancy than women with normal pregnancies. ${ }^{78}$

Blood pressure measurement is a screening test routinely used in antenatal care to detect or predict hypertensive disease. ${ }^{2}$ Accurate prediction of women at risk for pre-eclampsia is crucial to judicious allocation of monitoring resources and use of preventive treatment, ${ }^{9}$ with the prospect of improving maternal and neonatal outcome. Studies investigating the predictive accuracy of blood pressure measurement report conflicting results. In view of these conflicting reports it is uncertain whether blood pressure measurement should be used routinely as a predictive test or used only to diagnose hypertensive disorders in pregnancy once they are suspected. We carried out a systematic review to investigate the accuracy of blood pressure measurement for prediction of pre-eclampsia in pregnant women.

\section{METHODS}

This review was based on our previously published protocol. ${ }^{10}$ Librarians carried out two electronic searches of Medline, Embase, the Cochrane Library (2006;4), and Medion (www.mediondatabase.nl/) from inception to February 2007. The search strategy (see bmj.com) consisted of MeSH or key terms related to the disease (pre-eclampsia) and population (pregnant women) combined with methodological filters for identification of studies on diagnostic tests and causal 
Potentially relevant citations identified from electronic searches to capture primary articles on all tests used in prediction of pre-eclampsia $(n=19548)$

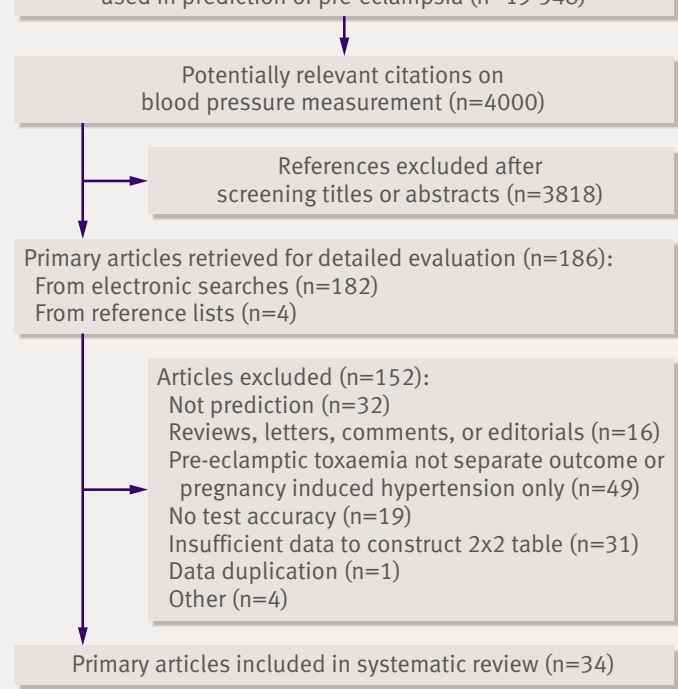

Fig 1| Literature identification and study selection

factors. ${ }^{11-13}$ A second search strategy was done to ascertain retrieval of relevant papers because "blood pressure" is a term related to both our index test of blood pressure measurement and the outcome preeclampsia. We checked reference lists of relevant studies to identify cited articles not captured by electronic searches and contacted authors of primary studies who had email addresses available. No language restrictions were applied. We included studies that reported on any technique to measure blood pressure in pregnant women in any healthcare setting and of any level of risk for pre-eclampsia. We included test accuracy studies allowing generation of $2 \times 2$ tables.

Trained reviewers independently screened titles and abstracts for relevance (JSC and KCV) and full papers for inclusion and data extraction (JSC and NdV). An explanation of extracted clinical, methodological, and statistical data has been published. ${ }^{10}$ Studies were assessed by one reviewer (JSC) for methodological quality against the quality assessment of diagnostic accuracy studies (QUADAS) criteria ${ }^{14}$ and randomly checked by a second reviewer $(\mathrm{NdV})$. How we assessed items is summarised on bmj.com. We also assessed application of preventive treatment. For multiple publications of one dataset we included only the most recent or complete study. Disagreements were resolved by consensus or a third reviewer.

Reference standards for pre-eclampsia were a persistent systolic blood pressure of $140 \mathrm{~mm} \mathrm{Hg}$ or more or a persistent diastolic blood pressure of $90 \mathrm{~mm}$ $\mathrm{Hg}$ or more, or both, with proteinuria of $0.3 \mathrm{~g} /$ day or more or a dipstick result of + or more $(30 \mathrm{mg} / \mathrm{dl}$ in a single urine sample), or both, new after 20 weeks of gestation. Severe pre-eclampsia was defined as systolic blood pressure of $160 \mathrm{~mm} \mathrm{Hg}$ or more or diastolic blood pressure of $110 \mathrm{~mm} \mathrm{Hg}$ or more, or both, with proteinuria $2.0 \mathrm{~g} /$ day or more or a dipstick result of ++ + or more, or both, of early onset ( $<34$ weeks) gestation. Superimposed pre-eclampsia was defined as the development of proteinuria of $0.3 \mathrm{~g} /$ day or more or a dipstick result of + or more after 20 weeks of gestation in chronically hypertensive women. Chronic hypertension was defined as hypertension present before pregnancy or detected before 20 weeks' gestation and not resolving within three months after delivery. ${ }^{15}$

\section{Data synthesis}

From the $2 \times 2$ tables we calculated sensitivity and specificity and plotted their results in receiver operating characteristic plots. We pooled results among groups of studies measuring similar blood pressure variables (systolic, diastolic, mean arterial, or increase) and similar outcome. We used a bivariate regression model that takes into account the negative correlation between sensitivity and specificity. This method has been extensively described elsewhere and is recently recommended for meta-analysis of diagnostic tests. ${ }^{16}{ }^{17}$ Briefly, rather than using a single outcome measure for each study, such as the diagnostic odds ratio, the bivariate model preserves the two dimensional nature of diagnostic data in a single model. This model incorporates the correlation that may exist between sensitivity and specificity within studies owing to possible differences in threshold between studies. When necessary the bivariate model uses a random effects approach for both sensitivity and specificity, allowing for heterogeneity beyond chance due to clinical or methodological differences between studies. In addition the model acknowledges the difference in precision by which sensitivity and specificity have been measured in each study. This means that studies with a larger number of women with pre-eclampsia receive more weight in the calculation of the pooled estimate of sensitivity, whereas studies with more women without pre-eclampsia are more influential in the pooling of specificity. To estimate a summary receiver operating characteristic curve and an area under that curve, we used the results of the model with the smallest Akaike's information criterion (a measure of the goodness of fit of an estimated statistical model). ${ }^{18}$ This model best accounts for heterogeneity between studies. We calculated areas under the curves in the first trimester for the complete summary receiver operating characteristic curve, which meant extension beyond the available data. We also calculated pooled sensitivities and specificities and derived likelihood ratios thereof. ${ }^{19}$

We carried out the following subgroup analyses, defined a priori: outcome (severe pre-eclampsia), sample (low risk sample $v$ high risk sample), number of readings (multiple measurement $v$ single measurement), and gestational age at testing (first trimester $v$ second trimester). Sensitivity analyses were done for application of preventive treatment and study quality. We considered studies of high quality when they scored positive on at least four of the following items: prospective design with consecutive recruitment, 
appropriate reference standard, follow-up greater than $90 \%$, adequate description of the index test, and reporting of preventive treatment.

All statistical analyses were done using SAS 9.1 for Windows (Proc NLMixed in the bivariate model). We used STATA/SE 9.00 to draw the receiver operating characteristic plots.

\section{RESULTS}

Figure 1 summarises the flow of studies through the review. Overall, 34 studies $^{\text {w1-w34 }}$ screening 60599 women (3341 pre-eclamptic women) were included, which comprised 28 cohorts, three randomised controlled trials, and three case-control studies resulting in $1352 \times 2$ tables. None of 12 authors contacted about unclear information in their articles provided additional information. The main reason given was "data not available." Twenty eight studies were prospective and six retrospective. The risk profile of the women included in the studies are on bmj.com. Sample sizes ranged from 22 to 22582 women. The incidence rates for pre-eclampsia ranged from $0.8 \%$ to $40.8 \%$ (median incidence 6.3\%; case-control studies excluded). Seven studies reported on "high risk" women (range for incidence rates $2.0 \%$ to $28.7 \%$ ). Since the criteria for high risk varied from women with an abnormal uterine artery detected by Doppler ultrasonography to women with chronic hypertension or diabetes, this may have caused the great variation in incidence. Fourteen studies explicitly reported exclusion of women with chronic hypertension and four studies reported inclusion of women with chronic hypertension (women at high risk). Twenty eight studies reported on blood pressure measurement in the second trimester. Eighteen studies reported on mean arterial pressure, defined as (diastolic blood pressure+one third $\times$ (systolic blood pressure-diastolic blood pressure) or automated mean arterial pressure readings. Six studies reported on systolic blood pressure; 11 on diastolic blood pressure; and three on increase of systolic blood

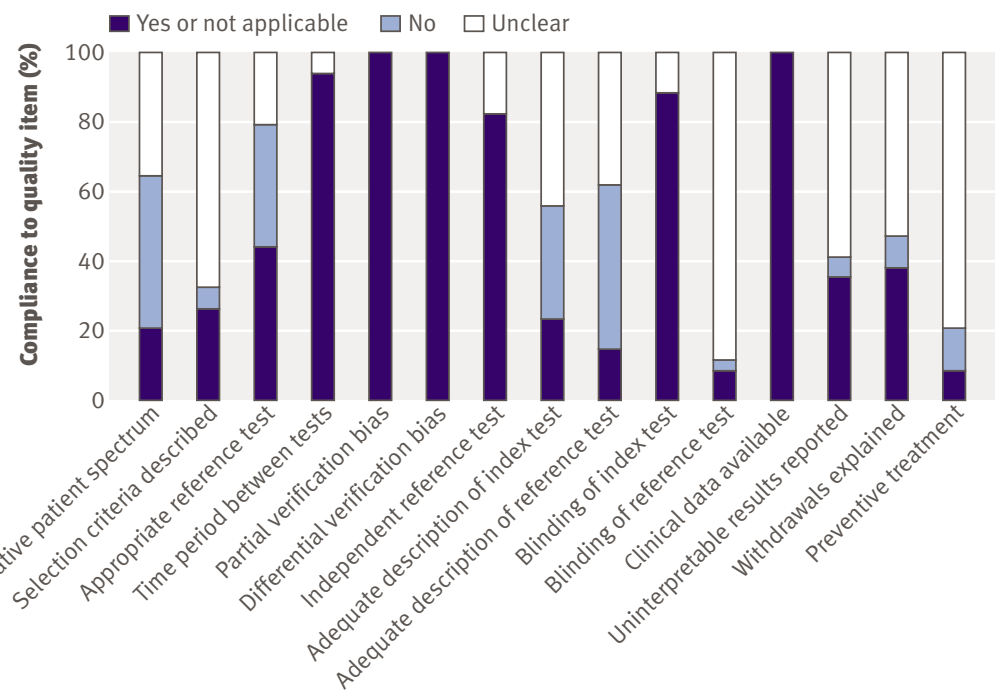

Fig 2 | Summary of quality assessment pressure or increase of diastolic blood pressure, or both. One study ${ }^{\text {w3 }}$ reported on diastolic midline estimating statistic of rhythm (MESOR) and two studies ${ }^{\text {w28 w31 }}$ reported on absolute blood pressure levels. Since both randomised controlled trials in women at low risk investigated calcium supplementation, which has not been proved effective, ${ }^{20}$ and one only included a placebo group, we analysed the trials together with the cohort studies. Four studies were excluded from the final meta-analysis (three casecontrol studies $^{\mathrm{w} 2 \mathrm{w} 15 \mathrm{w} 20}$ to further enhance validity and one study without a threshold for the index test ${ }^{\text {w26}}$ ).

\section{Quality assessment}

Figure 2 summarises the results of quality assessment. More than $70 \%$ of studies met the following items for quality assessment: period between tests, avoidance of partial and differential verification, independent reference test, blind assessment of index test, and clinical data available. Less than $30 \%$ of studies scored positive on the items for adequate patient spectrum; adequate descriptions of selection criteria, index test, and reference test; and blind assessment of reference test. Seven publications reported on the application of preventive treatment.

\section{Data analysis}

Figure 3 shows the receiver operating characteristic plots for the results for systolic blood pressure, diastolic blood pressure, mean arterial pressure, and increases of systolic blood pressure or diastolic blood pressure according to risk and trimester. Some studies are shown several times with several thresholds. Figure 4 shows the summary receiver operating characteristic curves for systolic blood pressure, diastolic blood pressure, and mean arterial pressure in low risk populations in the second trimester. The area under the curve was 0.68 (95\% confidence interval 0.64 to 0.72$)$ for systolic blood pressure, 0.66 (0.59 to 0.72 ) for diastolic blood pressure, and 0.76 (0.70 to 0.82$)$ for mean arterial pressure. For a specificity of $90 \%$ the sensitivities of diastolic blood pressure and mean arterial pressure were both $35 \%$, whereas for systolic blood pressure the sensitivity was only $24 \%$. For the six studies with a mean arterial pressure threshold of $85 \mathrm{~mm} \mathrm{Hg}$ or more the pooled sensitivity was $52 \%$ (95\% confidence interval $28 \%$ to $75 \%$ ) and pooled specificity $84 \%$ (95\% confidence interval $75 \%$ to $94 \%$ ); derived positive likelihood ratio 3.3 (95\% confidence interval 2.2 to 4.3 ) and negative likelihood ratio 0.57 (95\% confidence interval 0.35 to 0.80 ). A mean arterial pressure of $90 \mathrm{~mm} \mathrm{Hg}$ or more showed a pooled sensitivity of $62 \%$ $(35 \%$ to $89 \%)$ and a pooled specificity of $82 \%(72 \%$ to $92 \%)$; derived positive likelihood ratio 3.5 (2.0 to 5.0 ) and negative likelihood ratio 0.46 ( 0.16 to 0.75$)$. The three studies on increase of systolic blood pressure or increase of diastolic blood pressure, or both, showed poor predictive accuracy (fig 3). Poor predictive accuracy was shown for an increase in systolic blood pressure or diastolic blood pressure compared with 


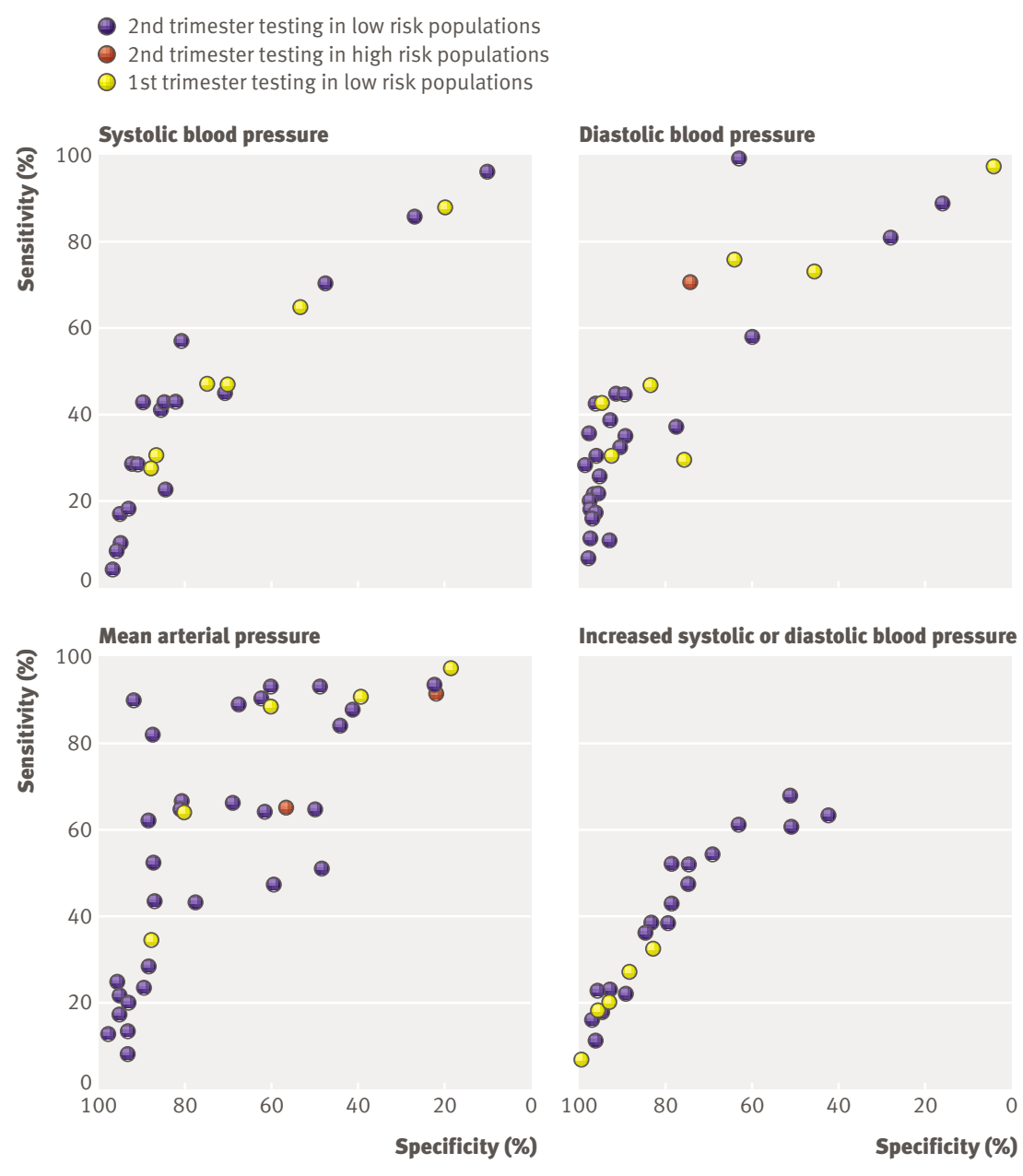

\begin{tabular}{l|l}
\hline Fig 3 & Receiver operating characteristic plots for all results according to timing of test during
\end{tabular} gestation
0.66 for diastolic blood pressure, and 0.76 for mean arterial pressure. Areas under the curves for blood pressure measurement in the first trimester were 0.66 for systolic blood pressure, 0.67 for diastolic blood pressure, and 0.79 for mean arterial pressure.

Studies that excluded women with chronic hypertension showed positive likelihood ratios for systolic blood pressure of 1.9 (95\% confidence interval 0.0 to 3.7 ), diastolic blood pressure 2.7 (0.0 to 5.5), and mean arterial pressure 2.6 (1.5 to 3.7). Negative likelihood ratios were, respectively, 0.6 ( 0.4 to 0.6$), 0.6$ (0.4 to 0.7 ), and 0.5 (0.3 to 0.8$)$.

Sensitivity analysis on preventive treatment was considered impractical because 25 studies (80\%) did not report on it. None of the studies met the criteria for high quality.

\section{DISCUSSION}

We reviewed the literature on the accuracy of blood pressure measurement during pregnancy to predict pre-eclampsia. Mean arterial pressure predicted preeclampsia fairly well (area under the curve between 0.70 and 0.80 ) whereas systolic blood pressure; diastolic blood pressure; and an increase of systolic blood pressure or increase of diastolic blood pressure, or both, predicted pre-eclampsia poorly (area under the curve $<0.70)$. In low risk populations a mean arterial pressure in the second trimester best predicted pre-eclampsia, with an area under the curve of 0.76 . An increased mean arterial pressure of $90 \mathrm{~mm} \mathrm{Hg}$ or more in the second trimester, however, showed a small increase in the likelihood of developing pre-eclampsia (positive likelihood ratio 3.5; negative likelihood ratio 0.46). In high risk populations a diastolic blood pressure of $75 \mathrm{~mm} \mathrm{Hg}$ or more at 13 to 20 weeks' gestation best predicted pre-eclampsia, although the accuracy of prediction was modest (positive likelihood ratio 2.8; negative likelihood ratio 0.39). Subgroup analyses were limited and did not improve predictive accuracy.

\section{Strengths and weaknesses of the review}

We carried out extensive literature searches without language restrictions, assessed the quality of the studies and the reporting, and used contemporary statistical methods. Many studies did not distinguish between pre-eclampsia and other hypertensive disorders in pregnancy (fig 1), nor did many report sufficient information to construct a $2 \times 2$ table. Contacting authors did not increase the number of included studies, resulting in a potential loss of relevant data. Quality assessment and subgroup analyses were hindered by unclear reporting in many studies, which is a common problem in diagnostic reviews. Previous studies reported that poor study design and conduct can affect estimates of diagnostic accuracy, ${ }^{2122}$ but it is not entirely clear how individual aspects of quality may affect this and to what magnitude in this particular area. Many strategies to account for differences in quality have been applied but none led to estimates that were systematically less optimistic than ignoring quality in 
meta-analyses of test accuracy studies. ${ }^{2324}$ As a result of unclear reporting it was not possible to carry out multivariate subgroup analysis on the basis of individual quality criteria, therefore we reported the overall results. Poor reporting occurred in details of the index test and reference standard, patient selection criteria, and blinding. Definitions of pre-eclampsia have changed over time, with previous definitions including oedema and increases in blood pressure. The measurement of blood pressure was poorly reported, underestimating the importance of recording diastolic blood pressure with Korotkoff phase $\mathrm{V}$ as this is more reliably recorded and more closely reflects direct measurement of diastolic blood pressure. ${ }^{25-27}$ Poor reporting of the device used underestimates the importance of its validation for blood pressure measurement during pregnancy. ${ }^{2829}$ Poor reporting of patient selection criteria may partly explain the great variability of incidence rates of pre-eclampsia not only between but within the categories of populations considered high risk or low risk. In some of the studies the population that was intended to be recruited differed from the population enrolled. The selection criteria for high risk varied from abnormal uterine artery detected by Doppler ultrasonography to pre-existent disease or mixtures thereof, such as chronic hypertension or diabetes. Large cohort studies (>300000 women) reflecting unselected populations, however, showed incidence rates of pre-eclampsia between $0.8 \%$ and $5.1 \%{ }^{30-32}$ This suggests that small studies of low risk or unselected populations within this review may have been prone to selection bias. Studies that tested in midtrimester should have taken into account that the predictive accuracy at 14 weeks may differ from that at 27 weeks because by definition no one develops preeclampsia in the first half of pregnancy.

Strengths and weaknesses in relation to other studies A published review in this area was restricted to evaluating risk factors for pre-eclampsia at the first antenatal visit. ${ }^{33}$ This review included four studies on blood pressure and concluded that the risk of preeclampsia was increased in women with a raised diastolic blood pressure $(>80 \mathrm{~mm} \mathrm{Hg})$ at the first antenatal visit (relative risk 1.48, 95\% confidence interval 1.0 to 1.9 ). Other published reviews did not apply appropriate methods for systematic reviews of screening tests or did not distinguish between different hypertensive disorders. ${ }^{34-39}$ Some of these reviews concluded that an increased mean arterial pressure ( $\geq 85 \mathrm{~mm} \mathrm{Hg}$ or $\geq 90 \mathrm{~mm} \mathrm{Hg}$ ) predicted transient hypertension rather than pre-eclampsia ${ }^{34-36}$ and that pregnant women with diastolic blood pressures of $70 \mathrm{~mm} \mathrm{Hg}$ or more or mean arterial pressures of $80 \mathrm{~mm}$ $\mathrm{Hg}$ or more in the second trimester have a small risk of developing pre-eclampsia. ${ }^{38}$

Unanswered questions, future research, and implications When deciding on whether a predictive test should be applied in clinical practice several things need to be considered: the prevalence of the disease and the predictive accuracy of the test, the cost of the test and its acceptability to patients, and the treatments available for the disease in question. Pre-eclampsia is a disease with relatively low prevalence. A clinically useful test would need to have a great area under the curve (preferably $>0.80$ ) or high positive likelihood ratio $(>10)$ and low negative likelihood ratio $(<0.10) .{ }^{40}$ From the results of this review mean arterial pressure still shows the greatest predictive accuracy in the first and second trimesters, with a relatively small likelihood ratio (positive likelihood ratio 3.5; negative likelihood ratio 0.46 ). In clinical practice measurement of mean arterial pressure at the first antenatal visit may improve the accuracy for estimating risk of pre-eclampsia. Although it will probably not make a clinical impact in isolation, it is highly likely that the prediction of preeclampsia will evolve through the development of algorithms that possibly include clinical, biophysical, and biochemical markers. Recently the authors of a large prospective study concluded that maternal variables, together with mean arterial pressure at 11 +0 to $13+6$ weeks, identify a group at high risk for preeclampsia. $^{41}$ Our data cannot rationalise current obstetrical practice of repeated blood pressure measurements during the first and second trimester in healthy women with a normal blood pressure at the first antenatal visit. A formal cost utility analysis is needed.

Women can experience unnecessary anxiety when being identified at risk of pre-eclampsia after an antenatal test. At present no pharmacological treatment or management strategy (for example, regular ultrasound scanning, early delivery) has been shown to effectively prevent the development of pre-eclampsia. Early antihypertensive treatment has been shown to only prevent severe hypertension, not any other complication. Research into aspirin as a treatment has, however, shown a modest preventive effect

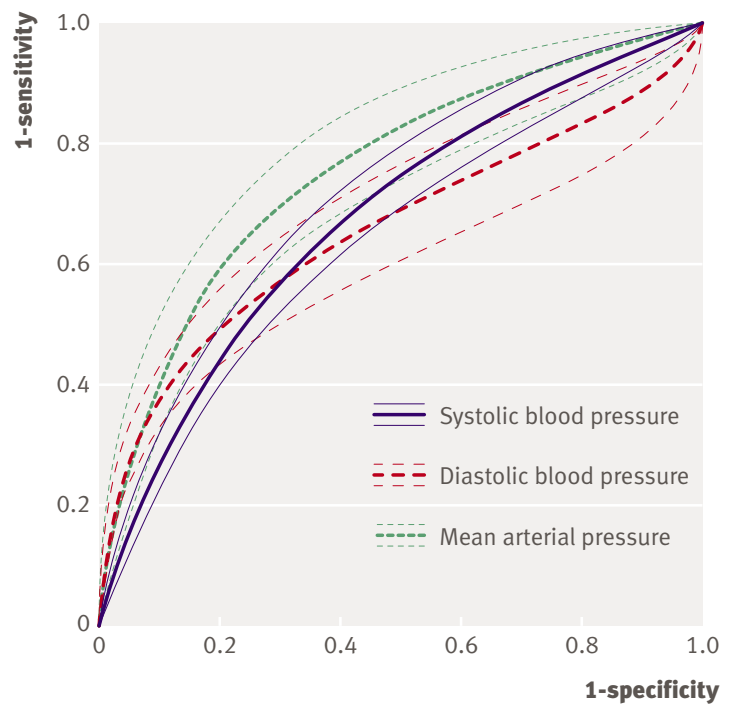

Fig 4 Summary receiver operating characteristic curves with $95 \%$ confidence intervals for systolic blood pressure, diastolic blood pressure, and mean arterial pressure in population of women at low risk of pre-eclampsia tested in second trimester 


\section{WHAT IS ALREADY KNOWN ON THIS TOPIC}

Pre-eclampsia is an important disorder in pregnancy, with potentially severe consequences for mother and child

Blood pressure measurement is routinely used in antenatal care to predict or detect hypertensive disorders

\section{WHAT THIS STUDY ADDS}

Mean arterial pressure is a better predictor for pre-eclampsia than systolic blood pressure, diastolic blood pressure, or increased blood pressure

Blood pressure measurements at the first antenatal visit for healthy normotensive women in the first and second trimester does not help predict pre-eclampsia

(relative risks of 0.9 for pre-eclampsia and 0.9 for fetal growth restriction ${ }^{42}$ ) in the absence of any serious side effects. Aspirin is a cheap and readily available preventive treatment. In this instance a false negative test result is potentially more harmful than a false positive test result.

It is imperative to differentiate between mild and severe disease because early or severe pre-eclampsia is associated with raised rates of maternal morbidity and mortality and has pronounced risks for the fetus, such as severe fetal growth restriction..$^{2-43}$ Future research should also concentrate on the development of algorithms that combine biochemical and biophysical markers, including blood pressure measurement-a diagnostic process used in clinical care. These may help improve the predictive accuracy of the tests to clinically important values.

We thank Faridi van Etten for help with the searches, Kees Boer and Hans Wolf for comments on the project and manuscript, and Koos Zwinderman for help with statistical analyses.

Contributors: All authors conceived and designed the study and drafted and critically revised the manuscript. JSC, $\mathrm{KCV}$, and NdV extracted the data. JSC, NdV, KCV, and JAMvdP analysed and interpreted the data. JSC, $\mathrm{NdV}$, and JAMvdP did the statistical analysis. JSC and JAMvdP are guarantors

Funding: ISC is supported by a project grant from the Academic Medical Centre fellowships for clinical guidelines.

Competing interests: None declared.

Ethical approval: Not required.

Provenance and peer review: Not commissioned; externally peer reviewed.

1 Khan KS, Wojdyla D, Say L, Gulmezoglu AM, Van Look PF. WHO analysis of causes of maternal death: a systematic review. Lancet 2006;367:1066-74.

2 Sibai B, Dekker G, Kupferminc M. Pre-eclampsia. Lancet 2005;365:785-99.

3 Vatten LJ, Skjaerven R. Is preeclampsia more than one disease? Obstet Gynecol Surv 2004;59:645-6.

4 Sibai BM. Diagnosis and management of gestational hypertension and preeclampsia. Obstet Gynecol 2003;102:181-92.

5 Hauth JC, Ewell MG, Levine RJ, Esterlitz JR, Sibai B, Curet LB, et al. Pregnancy outcomes in healthy nulliparas who developed hypertension. Calcium for Preeclampsia Prevention Study Group. Obstet Gynecol 2000;95:24-8

6 Redman CW, Sargent IL. Latest advances in understanding preeclampsia. Science 2005;308:1592-4.

7 Easterling TR, Benedetti TJ, Schmucker BC, Millard SP. Maternal hemodynamics in normal and preeclamptic pregnancies: a longitudinal study. Obstet Gynecol 1990;76:1061-9.
8 Rang S, Wolf H, Van Montfrans GA, Karemaker JM. Serial assessmen of cardiovascular control shows early signs of developing preeclampsia. J Hypertens 2004;22:369-76.

9 Duley L, Henderson-Smart DJ, Knight M, King JF. Antiplatelet agents for preventing pre-eclampsia and its complications. Cochrane Database Syst Rev 2004;CD004659.

10 Cnossen JS, van der Post JA, Mol BW, Khan KS, Meads CA, Ter RG. Prediction of pre-eclampsia: a protocol for systematic reviews of test accuracy. BMC Pregnancy Childbirth 2006;6:29.

11 Yale University School of Medicine. Finding the evidence on PubMed. 2003. http://info.med.yale.edu/library/reference/publications/ pubmed/.

12 Bachmann LM, Coray R, Estermann P, Ter Riet G. Identifying diagnostic studies in MEDLINE: reducing the number needed to read. I Am Med Inform Assoc 2002;9:653-8.

13 Haynes RB, Wilczynski N, McKibbon KA, Walker CJ, Sinclair JC. Developing optimal search strategies for detecting clinically sound studies in MEDLINE. I Am Med Inform Assoc 1994;1:447-58.

14 Whiting P, Rutjes AW, Reitsma JB, Bossuyt PM, Kleijnen J. The development of QUADAS: a tool for the quality assessment of studies of diagnostic accuracy included in systematic reviews. BMC Med Res Methodol 2003;3:25.

15 Brown MA, Lindheimer MD, de Swiet M, Van Assche A, Moutquin JM. The classification and diagnosis of the hypertensive disorders of pregnancy: statement from the International Society for the Study of Hypertension in Pregnancy (ISSHP). Hypertens Pregnancy 2001;20:IX-XIV.

16 Harbord RM, Deeks JJ, Egger M, Whiting P, Sterne JA. A unification of models for meta-analysis of diagnostic accuracy studies. Biostatistics 2007;8:239-51.

17 Reitsma JB, Glas AS, Rutjes AW, Scholten RJ, Bossuyt PM, Zwinderman AH. Bivariate analysis of sensitivity and specificity produces informative summary measures in diagnostic reviews. J Clin Epidemiol 2005;58:982-90.

18 Akaike H. A new look at the statistical model identification. IEEE Trans Automat Contr 1974;19:716-23.

19 Zwinderman AH, Bossuyt PM. We should not pool diagnostic likelihood ratios in systematic reviews. Stat Med 2008;27:687-97.

20 Meads CA, Cnossen JS, Meher S, Juarez-Garcia A, Ter Riet G, Duley L, et al. Methods of prediction and prevention of pre-eclampsiasystematic reviews of accuracy and effectiveness literature with economic modelling. Health Technol Assess 2008;12:1-270.

21 Lijmer JG, Mol BW, Heisterkamp S, Bonsel GJ, Prins MH, van der Meulen JH, et al. Empirical evidence of design-related bias in studies of diagnostic tests. JAMA 1999;282:1061-6.

22 Rutjes AW, Reitsma JB, Di Nisio M, Smidt N, van Rijn JC, Bossuyt PM Evidence of bias and variation in diagnostic accuracy studies. CMA 2006;174:469-76.

23 Leeflang M, Reitsma J, Scholten R, Rutjes A, Dinisio M, Deeks J, et al. Impact of adjustment for quality on results of metaanalyses of diagnostic accuracy. Clin Chem 2007;53:164-72.

24 Whiting P, Harbord R, Kleijnen J. No role for quality scores in systematic reviews of diagnostic accuracy studies. BMC Med Res Methodol 2005;5:19.

25 Brown MA, Reiter L, Smith B, Buddle ML, Morris R, Whitworth JA. Measuring blood pressure in pregnant women-a comparison of direct and indirect methods. Am J Obstet Gynecol 1994;171:661-7.

26 Brown MA, Hague WM, Higgins J, Lowe S, McCowan L, Oats J, et al. The detection, investigation and management of hypertension in pregnancy: full consensus statement. Aust N Z J Obstet Gynaecol 2000;40:139-55.

27 Shennan A, Gupta M, Halligan A, Taylor DJ, deSwiet M. Lack of reproducibility in pregnancy of Korotkoff phase IV as measured by mercury sphygmomanometry. Lancet 1996;347:139-42.

28 Brown MA, Robinson A, Buddle ML. Accuracy of automated blood pressure recorders in pregnancy. Aust N Z J Obstet Gynaecol 1998;38:262-5.

29 Pomini F, Scavo M, Ferrazzani S, De Carolis S, Caruso A, Mancuso S There is poor agreement between manual auscultatory and automated oscillometric methods for the measurement of blood pressure in normotensive pregnant women. J Matern Fetal Med 2001;10:398-403.

30 Conde-Agudelo A, Althabe F, Belizan JM, Kafury-Goeta AC. Cigarette smoking during pregnancy and risk of preeclampsia: a systematic review. Am J Obstet Gynecol 1999;181:1026-35.

31 Sebire NJ, Jolly M, Harris JP, Wadsworth J, Joffe M, Beard RW, et al. Maternal obesity and pregnancy outcome: a study of 287,213 pregnancies in London. Int J Obes Relat Metab Disord 2001;25:1175-82

32 Sebire NJ, Jolly M, Harris J, Regan L, Robinson S. Is maternal underweight really a risk factor for adverse pregnancy outcome? A population-based study in London. Br J Obstet Gynaecol 2001;108:61-6 
33 Duckitt K, Harrington D. Risk factors for pre-eclampsia at antenatal booking: systematic review of controlled studies. $B M$ J 2005;330:565-7.

34 Chesley LC, Sibai BM. Clinical significance of elevated mean arterial pressure in the second trimester. Am J Obstet Gynecol 1988;159:275-9.

35 Conde-Agudelo A, Lede R, Belizan J. Evaluation of methods used in the prediction of hypertensive disorders of pregnancy. Obstet Gynecol Surv 1994;49:210-22.

36 Dekker GA, Sibai BM. Early detection of preeclampsia. Am J Obstet Gynecol 1991;165:160-72.

37 O'Brien WF. Predicting preeclampsia. Obstet Gynecol 1990;75:445-52.

38 O'Brien WF. The prediction of preeclampsia. Clin Obstet Gynecol 1992;35:351-64.
39 Visser W, Wallenburg HC. Prediction and prevention of pregnancyinduced hypertensive disorders. Baillieres Best Pract Res Clin Obstet Gynaecol 1999;13:131-56.

40 Deeks JJ, Altman DG. Diagnostic tests 4: likelihood ratios. BMJ 2004;329:168-9.

41 Poon LCY, Kametas NA, Pandeva I, Valencia C, Nicolaides KH. Mean arterial pressure at $11+0$ to $13+6$ weeks in the prediction of preeclampsia. Hypertension 2008;51:1-7.

42 Askie LM, Duley L, Henderson-Smart DJ, Stewart LA. Antiplatelet agents for prevention of pre-eclampsia: a meta-analysis of individual patient data. Lancet 2007;369:1791-8.

43 Report of the National High Blood Pressure Education Program Working Group on high blood pressure in pregnancy. Am J Obste Gynecol 2000;183:S1-22.

Accepted: 30 March 2008 